\title{
A Simple Approach to Engineer SERS Substrates and Plasmonic Thin Film
}

\author{
Nur Uddin Ahamad, ${ }^{1}$ Md. Al-Amin, ${ }^{1}$ and Anatoli Ianoul ${ }^{2}$ \\ ${ }^{1}$ Department of Chemistry, Shahjalal University of Science and Technology, Sylhet 3114, Bangladesh \\ ${ }^{2}$ Department of Chemistry, Carleton University, 1125 Colonel By Drive, Ottawa, ON, Canada \\ Correspondence should be addressed to Nur Uddin Ahamad; nur-che@sust.edu
}

Received 20 May 2014; Accepted 15 August 2014; Published 25 August 2014

Academic Editor: Raphael Schneider

Copyright (C) 2014 Nur Uddin Ahamad et al. This is an open access article distributed under the Creative Commons Attribution License, which permits unrestricted use, distribution, and reproduction in any medium, provided the original work is properly cited.

\begin{abstract}
Solid supported 2D assembly of silver nanocubes was fabricated by Langmuir-Blodgett technique and employed to investigate its surface enhanced Raman scattering (SERS) and surface enhance fluorescence (SEF) activities by detecting Rh6G in solution of varied concentrations, that is, $10^{-12} \mathrm{M}, 10^{-9} \mathrm{M}, 10^{-6} \mathrm{M}$, and $10^{-3} \mathrm{M}$. SERS was detected from a nanomolar concentration of Rh6G whereas SEF was detected from a picomolar concentration. Further, the substrate was subjected to thermal annealing to fabricate plasmonic thin film. The formation of thin film was followed by monitoring its surface plasmon resonance spectra and atomic force microscopic images. It was observed that the characteristic spectral peaks of silver nanocubes merged into a broad spectral band as the annealing time was increased and the intensity of the band decreased with the formation of thin film. The obtained result implies that thermal annealing could be a simple approach to create nanoscale gaps in SERS substrate and to engineer continuous thin film from the assembly of discrete nanoparticles.
\end{abstract}

\section{Introduction}

Plasmonic metal nanoparticles such as silver, gold, and copper are gaining interest due to their unique optical properties that find applications in numerous fields of technology [1-4]. Noble metal nanoparticles support surface plasmons, coherent oscillation of conduction band electrons, which can be excited by electromagnetic radiation to give rise to an intense electric field. Such phenomenon is called surface plasmon resonance (SPR) [5, 6]. At resonance condition, absorption of light by nanoparticles occurs which appears as absorption peaks in their absorption spectrum. The position of the peak is highly sensitive to the refractive index of the external medium in which nanoparticles are placed. The sensitivity of nanoparticle's absorption peaks is the basis of SPR based molecular sensing [7-9]. In this context, two classes of surface plasmon resonance (SPR) are applied in sensing devices, for example, surface plasmon polariton (SPPs) and localized surface plasmon resonance (LSPRs). LSPR involves excitation of surface plasmons localized on the surface of discrete nanopartcle. SPPs involve excitation of surface plasmons that propagate along the nanoscale surface of a thin film of plasmonic materials $[10,11]$. LSPR is employed to sense molecular events occurring in the vicinity of nanoparticle's surface as the evanescent electric field exists within a few nanometers $(\sim 20 \mathrm{~nm})$ of the surface [11-13]. However, SPPs are employed to sense molecular events in the bulk medium as the electric field exists around $200 \mathrm{~nm}$ away from the surface [12]. In addition, E-field generated by excitation of surface plasmon by light modifies the scattering and emission processes of a nearby dipole resulting in surface-enhanced Raman, fluorescence and secondharmonic generation phenomena $[14,15]$. When nanoparticles are assembled in ordered structures on solid supports, some unique phenomena are observed such as hot spots generation and phenoresonance $[16,17]$. For this reason assembly of well-defined and reproducible plasmonic nanostructures has appeared as an essential part to develop novel SERS active substrates and sensor platforms. Nanotechnology offers several strategies to assemble nanostructures in a $2 \mathrm{D}$ fashion. 
Electron beam lithography and nanosphere lithography are two commonly used techniques to create ordered assembly of nanostructures [18]. However, Langmuir-Blodgett (LB) technique has evolved as efficient tool for organizing nanoscale building blocks of different composition, morphologies, and sizes. Langmuir-Blodgett film can be used as nanobiotemplate for the crystallization of protein which is considered as a major achievement in the field of both structural and functional proteomics [19]. LB based crystallization of proteins provides highly stable protein crystals and allows their characterization at a nano- and subnanoscale dimensions. Therefore, LB-based crystallography can have a tremendous impact in the field of industrial and clinical/therapeutic applications, opening new perspectives of personalized medicine [20]. In addition, LB film can be used to develop inorganic and biological sensor for health and environmental application [21, 22]. Moreover, inorganic nanostructures can be assembled on solid supports which have both scientific and technological interests $[14,15]$. Song and El-Sayed-Ali studied aggregation and optical field enhancing properties of silver nanocubes substrates fabricated by LB technique [23]. They found that control of interparticle spacing in a NC monolayer is difficult. Moreover nanoscale interparticle distance in plasmonic nanostructures is crucial for creating effective SERS substrates as such nanoscale gaps produce huge electromagnetic field, also called hot spots. We as well as other groups reported tuning of nanoscale interparticle gaps in Langmuir-Blodgett film using phospholipids and polymermolecules [24-26]. But incorporation of inert molecules within the nanostructures assembly may interfere with the signals of analytes. Therefore, it is important to find a contamination free approach to engineer SERS substrates with controlled nanoscale interparticle gaps. In addition, optical fiber based plasmonic detection is gaining considerable attention as this technique requires small volume of samples and renders detection of analytes remotely [26-29]. This technique utilizes the propagating surface plasmon modes generated on the surface of nanoscale film of plasmonic materials. Generation of nanoscale metallic film on sensor surface especially on fiber is challenging. Bialiayeu et al. showed the formation of nanoscale gold film on optical fiber functionalized with gold seeds [30]. But this method involves multisteps chemical functionalization procedure. Such nanoscale film can be alternatively fabricated by thermal annealing of discrete nanoparticles deposited on solid surface.

In this paper we engineered nanoscale silver film by reducing the interparticle distance among silver nanocubes deposited on LB substrate. We demonstrated that thermal annealing of $2 \mathrm{D}$ assembly of nanocubes could be a simple alternative route to tune interparticle distance and fabricate nanoscale films.

\section{Materials and Methods}

2.1. Chemicals. Silver nitrate $\left(\mathrm{AgNO}_{3}, 99+\%\right)$, polyvinylpyrrolidone (PVP, MW 55,000), ethylene glycol (EG), and chloroform were purchased from Sigma-Aldrich and used as obtained. Hydrochloric acid ( $\mathrm{HCl}, 38 \%)$ and ethanol (95\%) were obtained from Anachemia and Brampton, respectively, and used without further purification. Poly (diallyldimethylammonium chloride) solution (PDADMAC; $35 \mathrm{wt} \%$ in water, $\mathrm{MW}<100,000$ ), poly (sodium-4-styrenesulfonate) (PSS; MW 70,000), Rhodamine 6G (Rh6G), and hydrogen peroxide $(30 \%)$ were received from Caledon.

2.2. Preparation of Nanocubes. A colloidal solution of silver nanocubes (NC) was synthesized by a modified polyol process developed by Wiley et al. [2]. In a typical synthesis, $5 \mathrm{~mL}$ EG was placed in a $100 \mathrm{~mL}$ triple necked round bottom flask and heated at $140^{\circ} \mathrm{C}$ for $1 \mathrm{~h}$ under stirring with Teflon coated magnetic stirring bar using a temperature-controlled silicon oil bath. While EG was heated, EG solutions containing $\mathrm{AgNO}_{3}(94 \mathrm{mM})$ and PVP $(147 \mathrm{mM})$ were prepared. One $\mathrm{mL}$ of $\mathrm{HCl}$ solution in $\mathrm{EG}(3 \mathrm{mM})$, prepared $30 \mathrm{~min}$ prior to completion of EG heating time, was added quickly. Ten minutes after the injection of $\mathrm{HCl}, 3 \mathrm{~mL}$ of both $\mathrm{AgNO}_{3}$ and PVP solutions were added simultaneously in the hot reaction mixture at a rate of $\sim 750 \mathrm{~mL} / \mathrm{min}$ using glass syringes. The resulting solution was allowed to heat for $26 \mathrm{~h}$ under reflux conditions. The final product was cooled to room temperature and subjected to further purification.

2.3. Purification of Colloidal Silver Nanocubes. The assynthesized colloidal nanocubes contained unreacted EG, excess of PVP, and nanorods as a by-product. EG and PVP were removed by diluting the colloidal solution with ethanol ( $1: 1$ by volume) followed by centrifugation at $12,000 \mathrm{rpm}$. The precipitate was collected and redispersed in ethanol by sonication. This process was repeated 30 times for complete removal of EG and PVP. Silver nanorods were separated from the nanocubes by filtering the prewashed NC in ethanol using PTFE syringe filters (pore size: $0.45 \mu \mathrm{m}$ and $0.22 \mu \mathrm{m}$ ). The purified silver NC solution was further subjected to washing with chloroform by centrifugation (at least 3 times) prior to the LB film preparation. The final volume of the nanocube solution was $4 \mathrm{~mL}$.

2.4. Preparation of LB Monolayers. NIMA 311D trough, filled with Milli Q water $(18.2 \mathrm{~m} \Omega \mathrm{cm})$, was used to prepare the Langmuir monolayers of NC substrates. In order to create monolayers, $500 \mu \mathrm{L}$ of colloidal NC suspended in chloroform was injected onto the water subphase using a microsyringe. Monolayer was left for $30 \mathrm{~min}$ to allow chloroform evaporation. Surface pressure of the monolayer was measured with a paper Wilhelmy plate. Before transferring the monolayer onto solid supports several isotherm cycles were performed to anneal the films. Monolayer was transferred onto quartz substrates, cleaned with piranha solution and subsequently with a mixture of chloroform and methanol, at a surface pressure of $5 \mathrm{mN} / \mathrm{m}$.

2.5. UV-Vis Measurement. UV-vis spectra of the colloidal nanocubes in ethanol and of monolayers deposited on the quartz substrates were recorded using Shimadzu, UV-2450 $\mathrm{UV}$-vis spectrophotometer. 
2.6. Annealing of Glass Supported Silver Nanocubes Substrate. Glass supported silver nanocubes substrate was annealed by placing substrate in laboratory oven at $110^{\circ} \mathrm{C}$ for up to $221 \mathrm{hrs}$.

2.7. Topography Measurements. The topography of the NC monolayer, transferred onto quartz substrates at $5 \mathrm{mN} / \mathrm{m}$ surface pressure, was obtained using an Ntegra (NTMDT, Russia) atomic force microscope in semicontact mode in air at $23^{\circ} \mathrm{C}$ with $512 \times 512$ points per image. A $100 \times 100 \mu \mathrm{m}^{2}$ scanner (Ntegra) and cantilevers with rotated monolithic silicon tips (125 $\mu \mathrm{m}$-long, $40 \mathrm{~N} / \mathrm{m}$ spring constant Tap 300Al, resonance frequency $315 \mathrm{kHz}$, Budget Sensors) were used for all topographic measurements. The typical scan rate was $0.5 \mathrm{~Hz}$. AFM images were further processed by Nova image processing software.

2.8. SERS Measurement. Measurements of SERS spectra were performed using a single grating monochromator (Jobin Yvon, focal length $640 \mathrm{~mm}$ ) equipped with a liquid nitrogen cooled CCD camera (Princeton Instruments) and a notch filter to remove the excitation wavelength. Argon/Krypton ion laser system (Coherent), operating at $488 \mathrm{~nm}$ excitation wavelength with an output power of $20 \mathrm{~mW}$, was used for excitation. The spectral resolution of the instrument was $\sim 4 \mathrm{~cm}^{-1}$. The accumulation time was $60 \mathrm{~s}$. Final SERS spectrum of each substrate was the average of five spectra collected in different areas of the sample. The SERS spectra were collected by WinSpec/32 software and further processed by GRAMS/AI spectral data processing software.

2.9. Deposition of Polyelectrolyte (PE) Multilayers. PDADMAC and PSS bilayers were deposited on the nanostructure by self-assembly process [31]. Briefly, nanostructure consisting of nanocubes was immersed in a PDADMAC solution $(1 \mathrm{mg} / \mathrm{mL})$ and then into a PSS solution $(1 \mathrm{mg} / \mathrm{mL})$. In this way a total of 5 bilayers were deposited. Deposition of PE was monitored by PSS absorption intensity at $227 \mathrm{~nm}$.

2.10. Incorporation of Rh6G on the Nanostructure. Rh6G solutions having different concentrations, that is, $\mathrm{pM}, \mathrm{nM}$, $\mu \mathrm{M}$, and $\mathrm{mM}$, were prepared at a $\mathrm{pH}$ of 2.5 and the nanostructure containing PE (PSS on the top) was incubated into those solutions for $10 \mathrm{~min}$ followed by rinsing with copious of milliQ water. The substrates were dried under $\mathrm{N}_{2}$ flow and subjected to Raman measurement immediately.

\section{Results and Discussion}

3.1. Optical Properties of Silver Nanocubes. In this present work, a colloidal solution of silver nanocubes was synthesized by polyol reduction method as described earlier [32]. It is well-reported that plasmon response is sensitive to the size, morphology, and material of nanostructure as well as refractive index of the medium [33]. Both theoretical and experimental studies revealed that polyhedral shaped silver nanocrystals with well-defined facets and corners exhibit distinct optical signatures due to the excitations of different modes of localized surface plasmons (LSPs) [34].

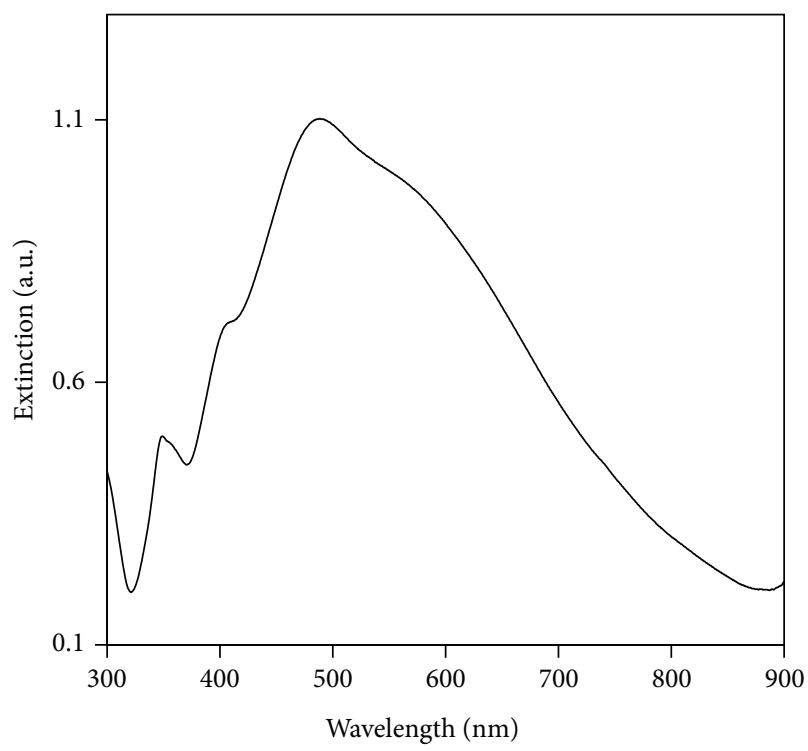

FIGURE 1: UV-vis extinction spectrum of colloidal silver nanocubes in ethanol.

Figure 1 shows the characteristic extinction spectrum of the as-synthesized colloidal silver nanocubes in ethanol. It is obvious that the spectrum consisted of four distinct peaks appearing at $600 \mathrm{~nm}, 500 \mathrm{~nm}, 420$, and $350 \mathrm{~nm}$. The first and second peaks have been assigned to dipolar and quadrupolar modes of LSP, respectively, whose amplitudes are stronger at the corners of the cube. The other two peaks, arising from multipolar modes, have higher amplitudes at the centre of the faces $[2,26,30,32,33]$. The size and shape of the nanocubes were confirmed by scanning electron microscopic images (SEM) as shown in Figure 2. It reveals the as-synthesized silver nanocube had sharp edge with a nominal edge length of $80 \mathrm{~nm}$. Although, occasionally, there are some nanorods, numerous rounds of centrifugation and filtration resulted in nanorods free monodisperse nanocube samples. The number and position of the spectral peaks of the purified NCs sample corroborated with the literature [26].

3.2. Engineering 2D Assembly of Supported NCs. LangmuirBlodgett (LB) technique has evolved as a powerful tool to fabricate organized assembly of nanoscale building blocks onto solid supports. In this present work, 2D assembly of silver nanocubes was engineered by transferring water supported Langmuir monolayer of nanocubes onto quartz substrates. Because of PVP coating, the as-synthesized NCs rendered hydrophobic surface which enabled them to spread and subsequent formation of monolayer on water subphase of LB trough. The packing and density of NCs in monolayer can be tuned by varying the surface pressure of the film which can be accomplished by compressing or expending the barrier of the trough. During the compression, the film undergoes different phase transitions which can be identified from the slope of the pressure-area isotherm. Figure 3 shows a typical pressure-area isotherm of silver NCs monolayer reflecting different phase transitions that it underwent from 


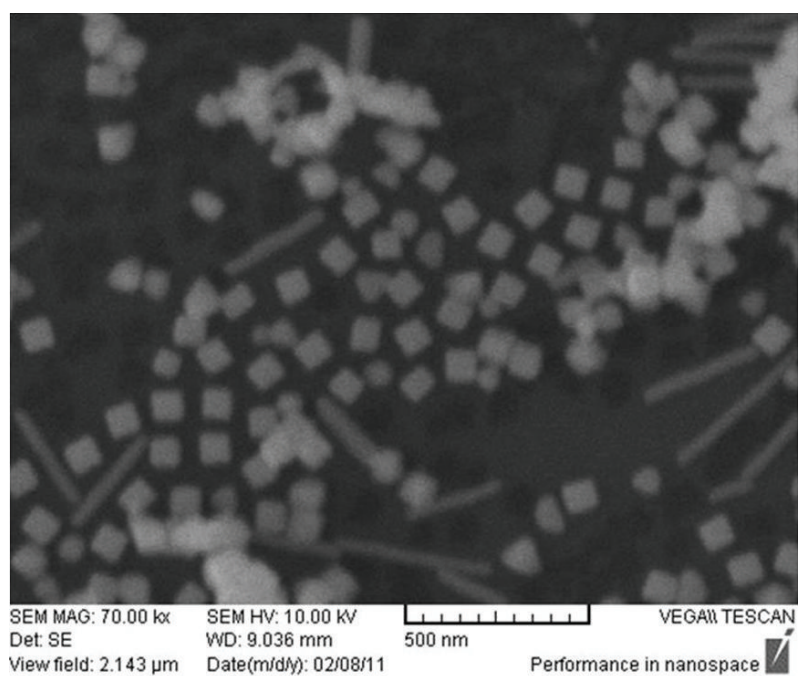

FIGURE 2: SEM image of silver nanocubes.

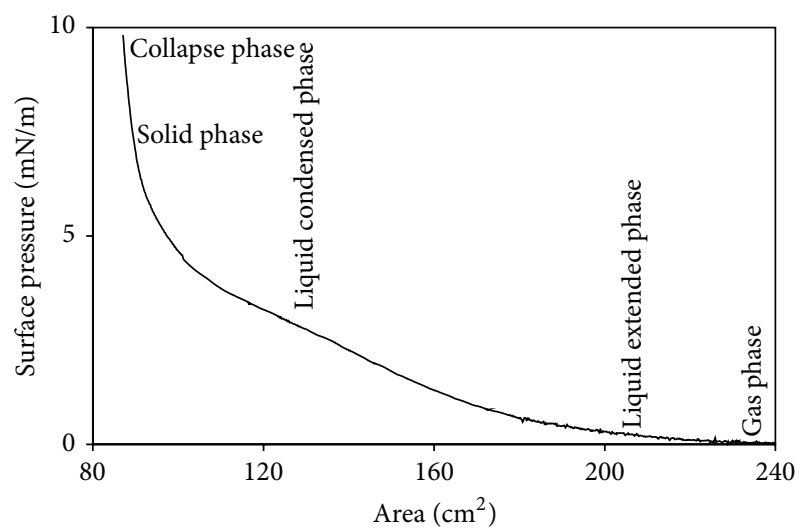

Figure 3: Pressure-area isotherm of the Langmuir monolayer consisted of silver nanocubes.
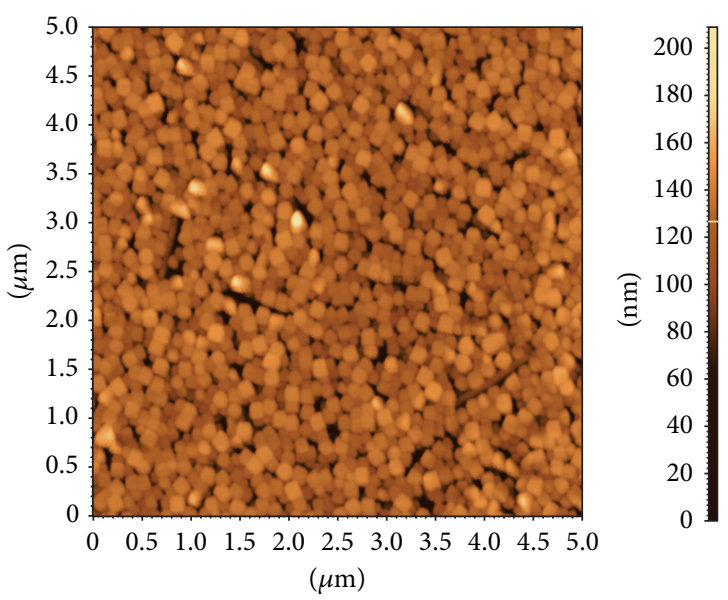

FIGURE 4: AFM topographic image of Langmuir-Blodgett (LB) film, transferred at a surface pressure of $5 \mathrm{mN} / \mathrm{m}$, consisted of silver nanocubes.

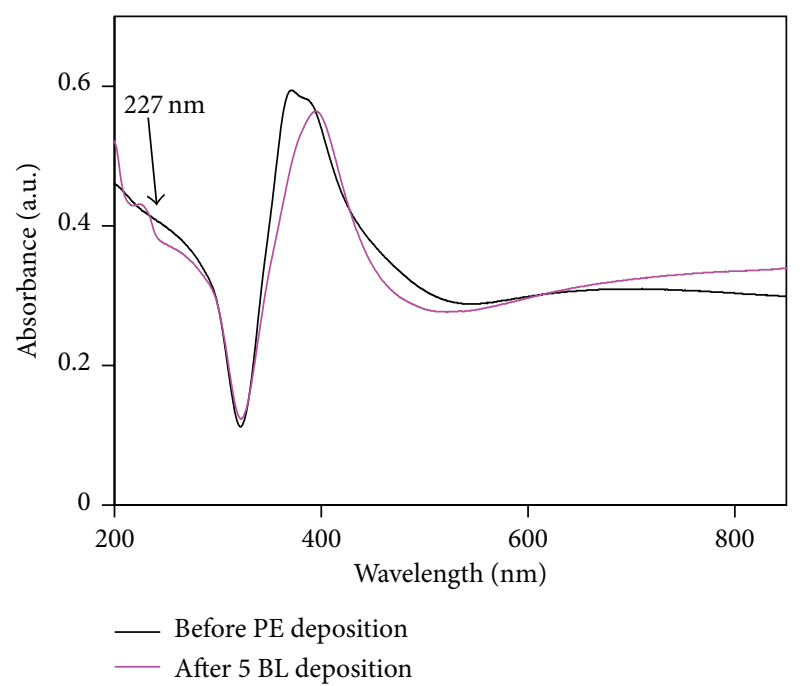

FIGURE 5: UV-vis absorption spectra showing adsorption of polyelectrolytes layers (PSS/PDADMAC) on the top of the solid supported silver nanocubes monolayer.

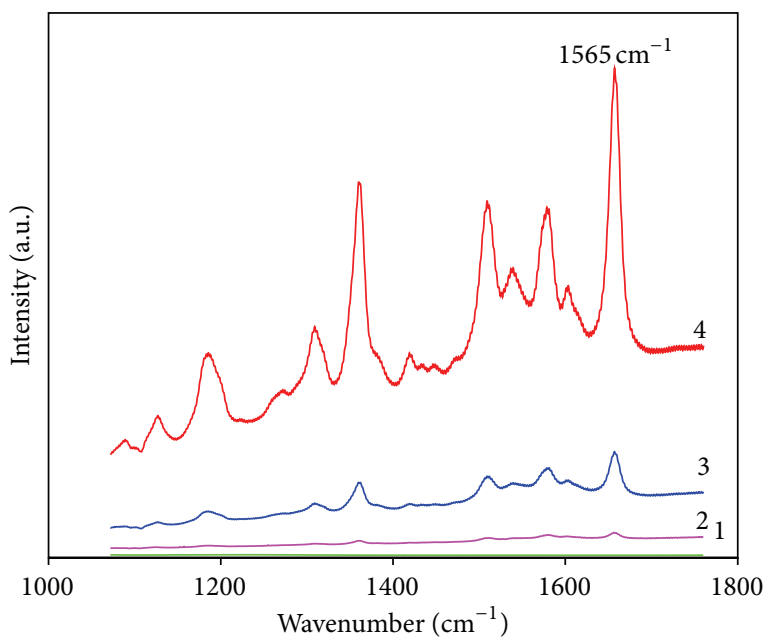

FIGURE 6: SERS spectra of silver nanocubes substrate (LB film) incubated in aqueous solutions of Rh6G having variable concentrations.

gas phase to solid phase through liquid condensed phase. In gas phase the NPs stay apart from each other such that surface density is low and therefore surface pressure does not change. In liquid phase, the NPs come close enough for mutual interaction to take place increasing the particle density which in turn increases the surface pressure. In solid phase, the NPs form close packing structure giving a sharp increase in surface pressure and after a critical surface pressure (collapse pressure) further compression collapses the monolayer. However, in case of NPs with anisotropic geometry like nanocubes, liquid phase consists of two subphases: a liquid extended (LE) phase where the NCs are close to each other but are in random orientations and liquid condensed (LC) phase where the NCs are orientated in a particular direction. Density of the particles increases upon increasing surface 


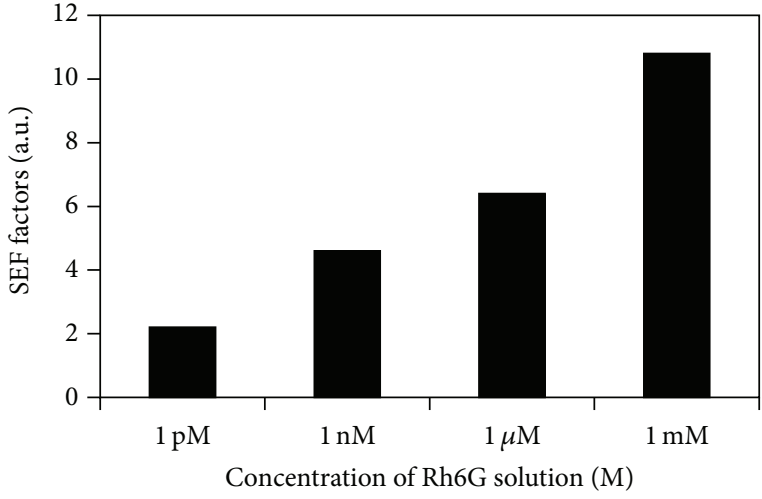

(a)

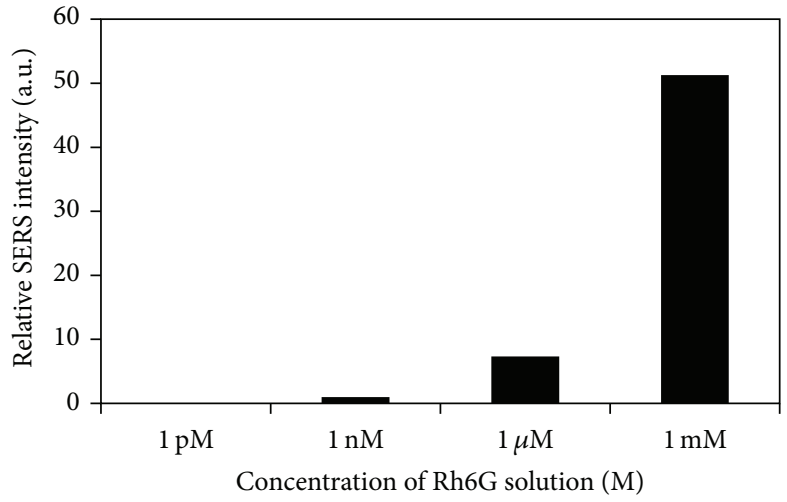

(b)

FIGURE 7: Relative intensities of (a) SERS and (b) SEF of silver nanocubes substrate $(5 \mathrm{mN} / \mathrm{m})$ incubated in Rh6G solutions having variable concentrations.

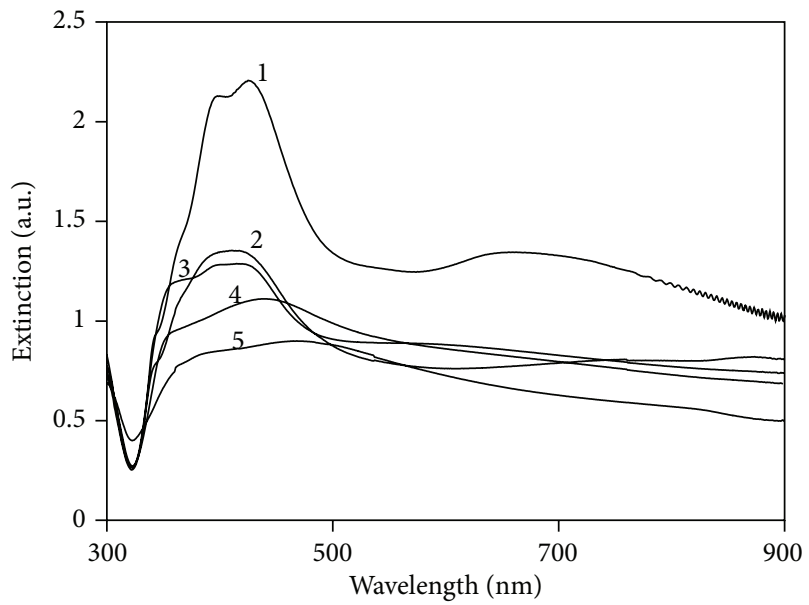

FIGURE 8: Extinction spectra of silver nanocubes substrate before annealing and after annealing at different annealing times.

pressure, brought about by film compression, which gives rise to the spectacular colour change of the Langmuir film.

Tapping mode AFM topographic image was taken in order to determine the topographic features of the LB film of NCs transferred at a surface pressure of $5 \mathrm{mN} / \mathrm{m}$ which correspond to a liquid condensed phase of the Langmuir film (Figure 4). It is obvious that the nanocubes were close to each other with nanoscale interparticle gaps and had face to face orientation. Such nanoscale gaps are known to enhance SERS signal $[35,36]$. However, there was a discontinuity within the monolayer which was because of the presence of impurity molecules in the water supported monolayer.

\subsection{Sensitivity of the Substrates toward Surface Enhanced} Raman Scattering (SERS) and Surface Enhanced Fluorescence $(S E F)$. Nanocubes with sharp edges have been proven to be a promising candidate for SERS substrates in molecular sensing with high sensitivity $[26,30,32]$. The intense EM field originating from sharp edges, faces and vertices amplifies the Raman and fluorescence optical signal. R6G, a strong fluorescent xanthene dye, was used as probe molecules for molecular detection purpose. To enhance adsorption of Rh6G five bilayers of PDADMAC/PSS were deposited on the top of nanocubes monolayer. Moreover, polyelectrolytes (PE) multilayer reduces the quenching of fluorescence by metal nanoparticles (NC). The attachment of the polyelectrolytes on nanocubes surfaces was confirmed by monitoring UVvis absorption signal intensity of PSS at $227 \mathrm{~nm}$ (Figure 5). The driving force behind the adsorption of PE on NC may be attributed to hydrogen bonding as well as dipole-ion interaction between the PVP and charged PE layer [31]. One issue with the nanocubes film upon incubation onto aqueous solutions is the stability of the film. We noticed some loss of nanocubes from the film which is believed to be because of the weak physisorbed NCs on the support. This was further confirmed by the fact that there was no significant intensity change in the SPR absorption band before and after incubation of nanocubes film onto aqueous solution. To investigate the SERS and SEF, four aqueous solutions of Rh6G with variable concentrations were prepared and NCs substrate was submerged into the solution. However, same nanocomposite substrate was incubated onto R6G solutions having different concentrations $\left(10^{-12} \mathrm{M}, 10^{-9} \mathrm{M}, 10^{-6} \mathrm{M}\right.$, and $\left.10^{-3} \mathrm{M}\right)$ starting from the lowest to the highest concentration. Such strategy reduces the concentration effect on the resultant SERS and SEF measurement. This strategy also eliminates the error arising from substrate preparation. Moreover, exposure to laser light decomposes the dye molecules into optically (Raman and fluorescence) inactive components. The Raman spectra were collected from at least five different spots of the substrate and finally averaged over using Grams AI software. Figure 6 shows the SERS spectra of the substrate incubated onto R6G solution of different concentrations. We could detect SERS from Rh6G at a concentration of $10^{-9} \mathrm{M}$. The band which appeared at $1650 \mathrm{~cm}^{-1}$, due to the $\mathrm{C}-\mathrm{C}$ stretching vibration of aromatic ring, was used as reference to determine SERS intensity of distance controlled $[31,37,38]$. In addition, SEF was observed from a solution concentration of $10^{-12} \mathrm{M}$. The enhancement factor was calculated as described below. 

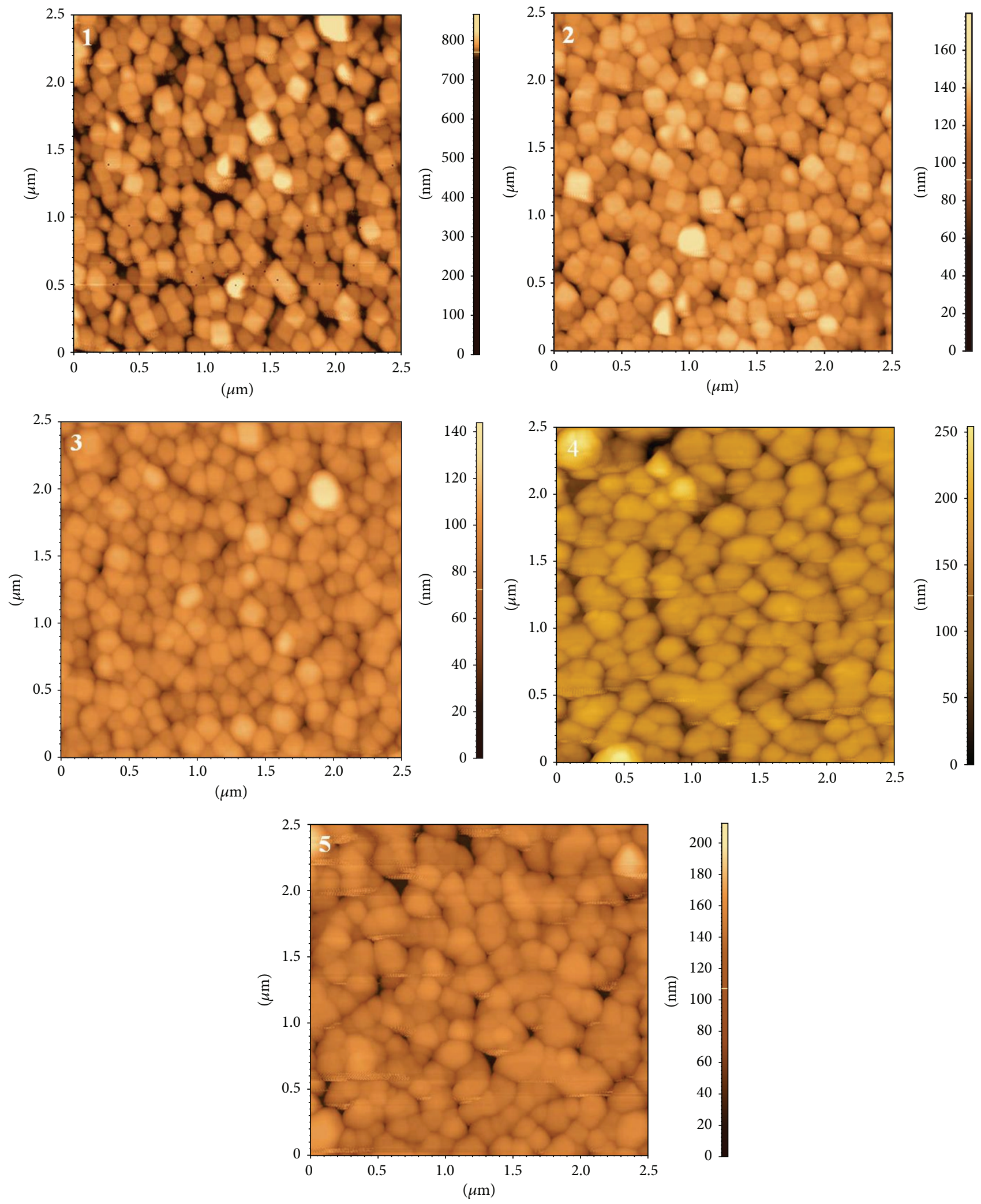

FIGURE 9: AFM topographic images (size of each image is $2.5 \mu \mathrm{m} \times 2.5 \mu \mathrm{m}$ ) of silver nanocubes monolayer recorded at different annealing times ((1) before annealing, (2) after $12 \mathrm{hrs,} \mathrm{(3)} \mathrm{after} 43 \mathrm{hrs,} \mathrm{(4)} \mathrm{after} 85 \mathrm{hrs,} \mathrm{and} \mathrm{(5)} \mathrm{after} 221 \mathrm{hrs).}$ 
The observed large enhancement factors for both SERS and SEF suggest that this monolayer can indeed serve as robust solid substrate for performing molecular detection with high sensitivity (Figure 7).

3.4. Enhancement Factor Calculation. Magnitude of optical signal enhancement in SERS or SEF is estimated by enhancement factor. Enhancement factor (EF) is defined by the following relation $\mathrm{EF}=I_{\mathrm{NC}} / I_{0}=I_{\mathrm{SERS}} / I_{\mathrm{RS}}\left(\right.$ or $\left.I_{\mathrm{SEF}} / I_{F}\right)$, where $I_{\mathrm{NC}}$ and $I_{0}$ are the intensities of Raman (or fluorescence) signal of Rh6G molecules adsorbed on Ag nanocubes substrate and that of on substrate without $\mathrm{Ag}$ nanocubes, respectively. $I_{\mathrm{SERS}}$ (or $I_{\mathrm{SEF}}$ ) and $I_{\mathrm{RS}}$ (or $I_{F}$ ) are the Raman (or fluorescence) intensities of the dyes under SERS (or SEF) and non-SERS (non-SEF) conditions, respectively. Relative intensity of SERS and fluorescence enhancement factors were calculated considering the substrate having the lowest intensity.

3.5. Fabrication of Film from Discrete NCs Assembly. Extinction spectrum of colloidal nanocubes in ethanol shows four peaks which are characteristics of $80 \mathrm{~nm}$ silver nanocubes. However, extinction spectrum of silver NCs deposited on glass shows four peaks along with a broad band at higher wavelengths. The broad band is because of coupling of interparticles plasmonic fields [38-41]. However, thermal annealing of the NCs substrate brought about two obvious spectral features, a decrease in the intensity of plasmon extinction peaks and merging of the peaks into a broad band (Figure 8). To elucidate the spectral change we investigated the morphology of the substrate after thermal annealing at different time intervals, that is, $12 \mathrm{hrs}, 43 \mathrm{hrs}, 85 \mathrm{hrs}$, and $221 \mathrm{hrs}$. The annealing temperature of the substrate was maintained at $110^{\circ} \mathrm{C}$, the highest temperature reached by our available laboratory oven. The morphology of the substrate at lower annealing time did not change significantly. Figure 9 shows the AFM images of the substrate before and after annealing at different time intervals. The AFM image of the substrate before annealing clearly shows the assembly of NCs on the glass support. As the annealing time was increased, truncation of the cubes' corners started and the interparticle gaps were reduced while at the same time the height of NCs decreased finally forming a continuous film. Therefore, the spectral change was brought about by the morphological change of the substrate. Before thermal annealing, due to the sharp edges and corners of nanocubes, all modes of surface plasmons are excited [41, 42]. Therefore the spectrum consisted of four peaks. As the annealing time was increased truncation of corners took place which diminished the excitation of multipolar plasmonic mode. Finally the discrete NCs formed a continuous film which supports propagating surface plasmons that can be excited at infrared region and the intensity of LSPR absorption was decreased and merging of the peaks into a broad band occurred [31, 41-45]. However, the annealing time can be drastically reduced by increasing the annealing temperature [46-48]. The resultant nanoscale film can be used to build up PSP based molecular sensing and temperature gradient detection [49-51].

\section{Conclusion}

In this work, we presented a simple approach to engineer effective SERS and SEF substrates using LB technique and showed that plasmonic thin film can be prepared by thermal annealing of LB film which consisted of metal nanoparticles with large interparticles gaps. The LB substrate exhibited SERS signal of Rh6G at nanomolar solution whereas SEF signal was observed at a concentration of picomolar solution. However formation of continuous thin film by thermal annealing of low density LB monolayer of metal nanoparticles can be traced by monitoring its surface plasmon resonance peaks. It has been observed that the spectral peaks of discrete silver nanocubes merge into a broad band with increasing annealing time and the intensity of the band diminishes when the film becomes continuous. Although, in this investigation, the annealing time was longer, it can be reduced by elevation of annealing temperature. Further research will be addressed on this issue.

\section{Conflict of Interests}

The authors declare that there is no conflict of interests regarding the publication of this paper.

\section{Acknowledgments}

Financial support was provided by NSERC, CFI, and Carleton University.

\section{References}

[1] P. K. Jain, X. Huang, I. H. El-Sayed, and M. A. El-Sayed, "Noble metals on the nanoscale: Optical and photothermal properties and some applications in imaging, sensing, biology, and medicine," Accounts of Chemical Research, vol. 41, no. 12, pp. 1578-1586, 2008.

[2] B. Wiley, Y. Sun, and Y. Xia, "Synthesis of silver nanostructures with controlled shapes and properties," Accounts of Chemical Research, vol. 40, no. 10, pp. 1067-1076, 2007.

[3] H. Wang, F. Tam, N. K. Grady, and N. J. Halas, "Cu nanoshells: effects of interband transitions on the nanoparticle plasmon resonance," The Journal of Physical Chemistry B, vol. 109, no. 39, pp. 18218-18222, 2005.

[4] A. F. Alvarez-Paneque, B. Rodríguez-González, I. PastorizaSantos, and L. M. Liz-Marzán, "Shape-templated growth of Au@Cu nanoparticles," Journal of Physical Chemistry C, vol. 117, no. 6, pp. 2474-2479, 2013.

[5] H. A. Atwater, “The promise of plasmonics., Scientific American, vol. 296, no. 4, pp. 56-63, 2007.

[6] E. Ozbay, "Plasmonics: merging photonics and electronics at nanoscale dimensions," Science, vol. 311, no. 5758, pp. 189-193, 2006.

[7] A. N. Shipway, E. Katz, and I. Willner, "Nanoparticle arrays on surfaces for electronic, optical, and sensor applications," ChemPhysChem, vol. 1, no. 1, pp. 18-52, 2000.

[8] J. Homola, "Present and future of surface plasmon resonance biosensors," Analytical and Bioanalytical Chemistry, vol. 377, no. 3, pp. 528-539, 2003. 
[9] H. J. Lee, Y. Li, A. W. Wark, and R. M. Corn, "Enzymatically amplified surface plasmon resonance imaging detection of DNA by exonuclease III digestion of DNA microarrays," Analytical Chemistry, vol. 77, no. 16, pp. 5096-5100, 2005.

[10] A. G. Brolo, "Plasmonics for future biosensors," Nature Photonics, vol. 6, no. 11, pp. 709-713, 2012.

[11] R. H. Ritchie, "Plasma losses by fast electrons in thin films," vol. 106, pp. 874-881, 1957.

[12] H. Knobloch, H. Brunner, A. Leitner, F. Aussenegg, and W. Knoll, "Probing the evanescent field of propagating plasmon surface polaritons by fluorescence and Raman spectroscopies," The Journal of Chemical Physics, vol. 98, no. 12, pp. 10093-10095, 1993.

[13] W. Knoll, "Interfaces and thin films as seen by bound electromagnetic waves," Annual Review of Physical Chemistry, vol. 49, no. 1, pp. 569-638, 1998.

[14] R. Wannemacher, "Plasmon-supported transmission of light through nanometric holes in metallic thin films," Optics Communications, vol. 195, no. 1-4, pp. 107-118, 2001.

[15] L. Yin, V. K. Vlasko-Vlasov, A. Rydh et al., "Surface plasmons at single nanoholes in Au films," Applied Physics Letters, vol. 85, no. 3, pp. 467-469, 2004.

[16] S. Coyle, M. C. Netti, J. J. Baumberg et al., "Confined plasmons in metallic nanocavities," Physical Review Letters, vol. 87, no. 17, Article ID 176801, 2001.

[17] C. Genet and T. W. Ebbesen, "Light in tiny holes," Nature, vol. 445, no. 7123, pp. 39-46, 2007.

[18] U. Huebner, H. Schneidewind, D. Cialla et al., "Fabrication of regular patterned SERS arrays by electron beam lithography," in Biophotonics: Photonic Solutions for Better Health Care II, vol. 7715 of Proceedings of SPIE, p. 771536, 2010.

[19] E. Pechkova, N. L. Bragazzi, and C. Nicoloni, "Advances in nanocrystallography as a proteomic tool," Advances in Protein Chemistry and Structural Biology, vol. 95, pp. 163-191, 2014.

[20] C. Nicolini, N. Bragazzi, and E. Pechkova, "Nanoproteomics enabling personalized nanomedicine," Advanced Drug Delivery Reviews, vol. 64, no. 13, pp. 1522-1531, 2012.

[21] N. L. Bragazzi, E. Pechkova, D. Scudieri, T. B. C. Terencio, M. Adami, and C. Nicolinia, "Recombinant laccase: II. Medical biosensor," Critical Reviews in Eukaryotic Gene Expression, vol. 22, no. 3, pp. 197-203, 2012.

[22] C. Nicolini, M. Adami, M. Sartore et al., "Prototypes of newly conceived inorganic and biological sensors for health and environmental applications," Sensors, vol. 12, no. 12, pp. 1711217127, 2012.

[23] Y. Song and H. E. Elsayed-Ali, "Aqueous phase Ag nanoparticles with controlled shapes fabricated by a modified nanosphere lithography and their optical properties," Applied Surface Science, vol. 256, no. 20, pp. 5961-5967, 2010.

[24] Z. Wei, W. Xu, W. Hu, and D. Zhu, "Langmuir-blodgett monolayer as an efficient $\mathrm{p}$-conducting channel of ambipolar organic transistors and a template for n-type molecular alignment," Langmuir, vol. 25, no. 6, pp. 3349-3351, 2009.

[25] P. Yang and F. Kim, "Langmuir-blodgett assembly of onedimensional nanostructures," ChemPhysChem, vol. 3, no. 6, pp. 503-506, 2002.

[26] N. Ahamad and A. Ianoul, "Using phospholipids to control interparticle distance in SERS-active substrates," The Journal of Physical Chemistry C, vol. 115, no. 9, pp. 3587-3594, 2011.
[27] C. Yuan, J. Furlong, P. Burgos, and L. J. Johnston, “The size of lipid rafts: an atomic force microscopy study of ganglioside GM1 domains in sphingomyelin/DOPC/cholesterol membranes," Biophysical Journal, vol. 82, no. 5, pp. 2526-2535, 2002.

[28] M. A. Mahmoud, C. E. Tabor, and M. A. El-Sayed, "Surfaceenhanced raman scattering enhancement by aggregated silver nanocube monolayers assembled by the Langmuir-Blodgett technique at different surface pressures," The Journal of Physical Chemistry C, vol. 113, no. 14, pp. 5493-5501, 2009.

[29] M. A. Mahmoud and M. A. El-Sayed, "Comparative study of the assemblies and the resulting plasmon fields of LangmuirBlodgett assembled monolayers of silver nanocubes and gold nanocages," Journal of Physical Chemistry C, vol. 112, no. 37, pp. 14618-14625, 2008.

[30] A. Bialiayeu, C. Caucheteur, N. Ahamad, A. Ianoul, and J. Albert, "Self-optimized metal coatings for fiber plasmonics by electroless deposition," Optics Express, vol. 19, no. 20, pp. 1874218753, 2011.

[31] Y. Saito, J. J. Wang, D. A. Smith, and D. N. Batchelder, "A simple chemical method for the preparation of silver surfaces for efficient SERS," Langmuir, vol. 18, no. 8, pp. 2959-2961, 2002.

[32] Y. Sun and Y. Xia, "Shape-controlled synthesis of gold and silver nanoparticles," Science, vol. 298, no. 5601, pp. 2176-2179, 2002.

[33] N. Ahamad, A. Bottomley, and A. Ianoul, "Optimizing refractive index sensitivity of supported silver nanocube monolayers," Journal of Physical Chemistry C, vol. 116, no. 1, pp. 185-192, 2012.

[34] C. Noguez, "Surface plasmons on metal nanoparticles: the influence of shape and physical environment," The Journal of Physical Chemistry C, vol. 111, no. 10, pp. 3606-3619, 2007.

[35] B. Gao, G. Arya, and A. R. Tao, "Self-orienting nanocubes for the assembly of plasmonic nanojunctions," Nature Nanotechnology, vol. 7, no. 7, pp. 433-437, 2012.

[36] A. Tao, P. Sinsermsuksakul, and P. Yang, "Polyhedral silver nanocrystals with distinct scattering signatures," Angewandte Chemie, vol. 45, no. 28, pp. 4597-4601, 2006.

[37] D. Pristinski, S. Tan, M. Erol, H. Du, and S. Sukhishvili, "In situ SERS study of Rhodamine 6G adsorbed on individually immobilized Ag nanoparticles," Journal of Raman Spectroscopy, vol. 37, no. 7, pp. 762-770, 2006.

[38] P. Hildebrandt and M. Stockhurger, "Surface-enhanced resonance Raman spectroscopy of Rhodamine 6G adsorbed on colloidal silver," The Journal of Physical Chemistry, vol. 88, no. 24, pp. 5935-5944, 1984.

[39] C. E. Reed, J. Giergiel, S. Ushioda, and J. C. Hemminger, "Effects of annealing on the attenuated-total-reflection spectra of coldevaporated silver films," Physical Review B, vol. 31, no. 4, pp. 1873-1880, 1985.

[40] P. Royer, J. P. Goudonnet, R. J. Warmack, and T. L. Ferrell, "Substrate effects on surface-plasmon spectra in metal-island films," Physical Review B, vol. 35, no. 8, pp. 3753-3759, 1987.

[41] G. Andreasen, P. L. Schilardi, O. Azzaroni, and R. C. Salvarezza, “Thermal annealing of patterned metal surfaces," Langmuir, vol. 18, no. 26, pp. 10430-10434, 2002.

[42] S. E. Roark, D. J. Semin, and K. L. Rowlen, "Quantitative evaluation of SERS-active Ag film nanostructure by atomic force microscopy," Analytical Chemistry, vol. 68, no. 3, pp. 473480, 1996.

[43] H. Bi, W. Cai, L. Zhang, D. Martin, and F. Träger, "Annealinginduced reversible change in optical absorption of Ag nanoparticles," Applied Physics Letters, vol. 81, no. 27, pp. 5222-5224, 2002. 
[44] I. Doron-Mor, Z. Barkay, N. Filip-Granit, A. Vaskevich, and I. Rubinstein, "Ultrathin gold island films on silanized glass. Morphology and optical properties," Chemistry of Materials, vol. 16, no. 18, pp. 3476-3483, 2004.

[45] D. J. Semin and K. L. Rowlen, "Influence of vapor deposition parameters on SERS active Ag film morphology and optical properties," Analytical Chemistry, vol. 66, no. 23, pp. 4324-4331, 1994.

[46] A. Feofanov, A. Ianoul, E. Kryukov et al., "Nondisturbing and stable SERS-active substrates with increased contribution of long-range component of Raman enhancement created by high-temperature annealing of thick metal films," Analytical Chemistry, vol. 69, no. 18, pp. 3731-3740, 1997.

[47] N. Ahamad, M. Al- Amin, and A. Ianoul, "Distance dependent surface enhanced Raman and fluorescence by supported 2D assembly of plasmonic metal nanoparticles," Asian Journal of Chemistry, vol. 25, no. 16, pp. 9226-9232, 2013.

[48] M. Litorja, C. L. Haynes, A. J. Haes, T. R. Jensen, and R. P. van Duyne, "Surface-enhanced Raman scattering detected temperature programmed desorption: optical properties, nanostructure, and stability of silver film over $\mathrm{SiO}_{2}$ nanosphere surfaces," The Journal of Physical Chemistry B, vol. 105, no. 29, pp. 69076915, 2001.

[49] J.-H. Yoo and S.-W. Lee, "Fabrication and characterization of quantum dots-bound hydrogels with fluorescent and temperature-sensitive functionalities," Journal of Nanoscience and Nanotecnology, vol. 14, no. 10, pp. 7648-7653, 2014.

[50] M. I. J. Stich, L. H. Fischer, and O. S. Wolfbeis, "Multiple fluorescent chemical sensing and imaging," Chemical Society Reviews, vol. 39, no. 8, pp. 3102-3114, 2010.

[51] L. Sun, J. Yu, H. Peng, J. Z. Zhang, L. Shi, and O. S. Wolfbeis, "Temperature-sensitive luminescent nanoparticles and films based on a terbium (III) complex probe," The Journal of Physical Chemistry C, vol. 114, no. 29, pp. 12642-12648, 2010. 

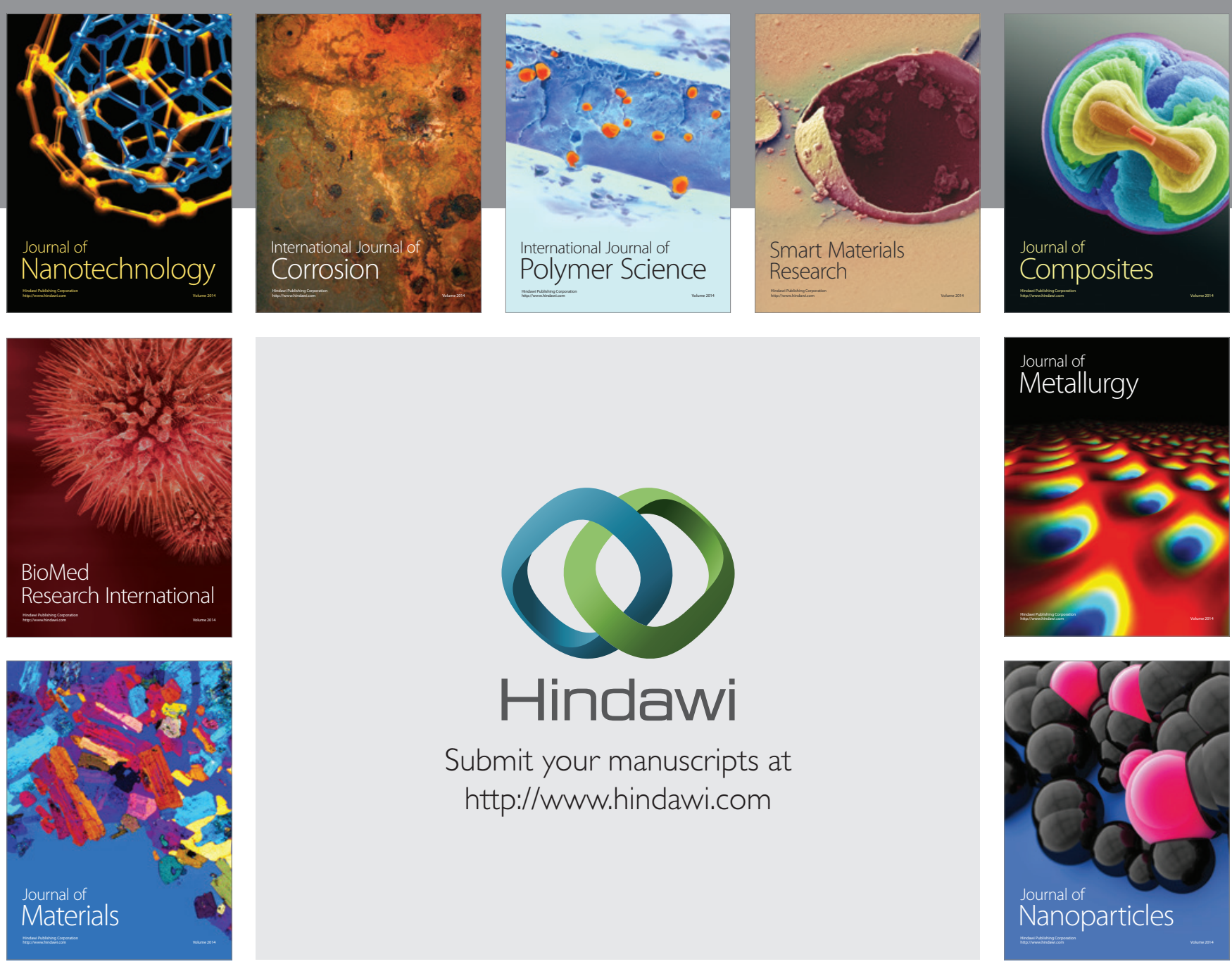

Submit your manuscripts at http://www.hindawi.com
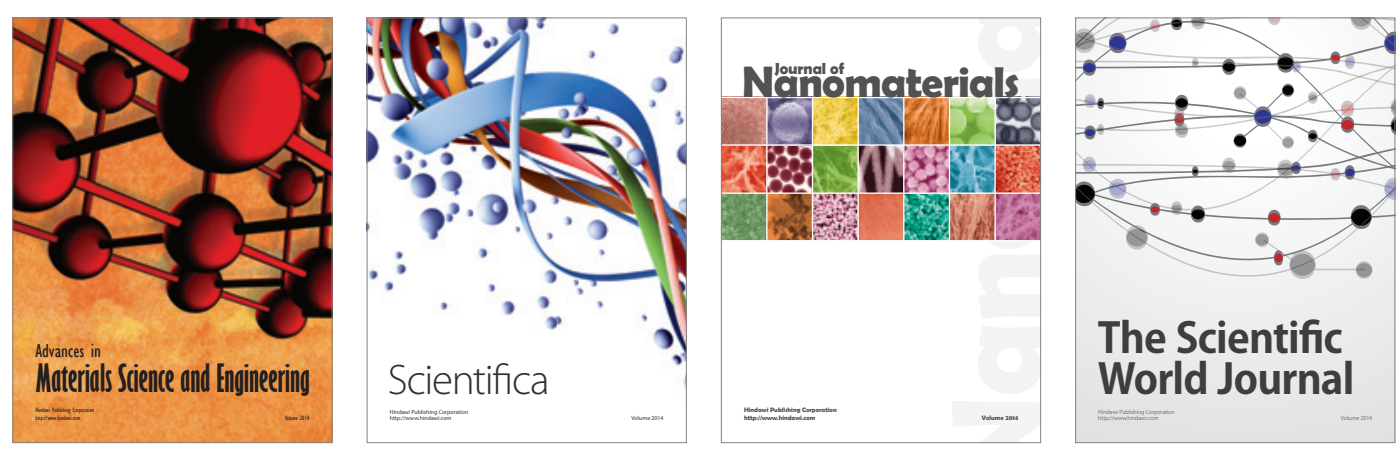

\section{The Scientific World Journal}
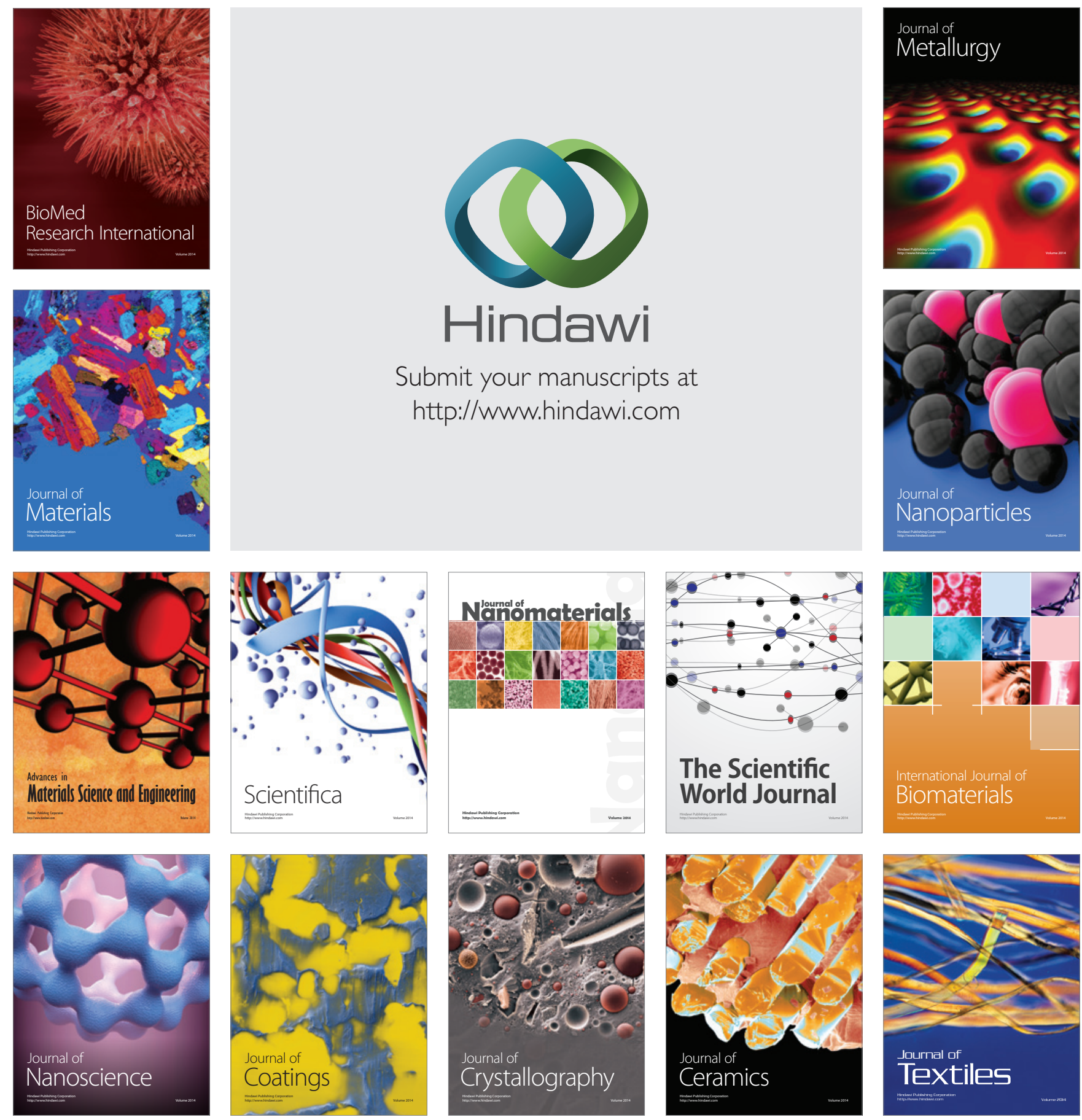